\title{
Erratum
}

\section{Multiphoton-Induced X-Ray Emission and Amplification from Clusters}

A. McPherson, T.S. Luk, B.D. Thompson, K. Boyer, C.K. Rhodes

Appl. Phys. B 57, 337 (1993)

Due to printing errors equations (6) and (9) of this paper are incorrect and should read:

$I_{0}=\frac{\varepsilon_{e}^{2}}{8 \pi \alpha n^{2 / 3} \hbar r_{0}^{2}}, \quad(n \geq 3)$,

$\frac{g_{\lambda}}{n_{e}}=\frac{\lambda_{x}^{3}}{8 \pi Z \sqrt{2}}\left(\frac{A_{2}}{c}\right)\left(\frac{\sigma_{e i}}{r_{0}^{2}}\right)\left(\frac{M c^{2} a_{0}}{e^{2}}\right)^{1 / 2}\left(\frac{r_{0}}{a_{0}}\right)^{1 / 2} \mathrm{~cm}^{2}$. 